\title{
Relationship between anti-acetylcholine receptor antibodies and the development of post-thymectomy myasthenia gravis in patients with thymoma: a single-center experience
}

\author{
Yusuke Nabe, Teppei Hashimoto, Kanji Tanaka, Yasuhiro Fujita, Katsuma Yoshimatsu, Yukiko Nemoto, \\ Rintaro Oyama, Hiroki Matsumiya, Masataka Mori, Masatoshi Kanayama, Akihiro Taira, \\ Shinji Shinohara, Taiji Kuwata, Masaru Takenaka, Yuko Tashima, Koji Kuroda, Fumihiro Tanaka \\ Second Department of Surgery, School of Medicine, University of Occupational and Environmental Health, Kitakyushu, Japan \\ Contributions: (I) Conception and design: H Matsumiya, M Mori, M Kanayama, M Takenaka; (II) Administrative support: T Hashimoto, Y Fukuichi, \\ A Taira; (III) Provision of study materials or patients: K Tanaka, Y Fujita, Y Tashima; (IV) Collection and assembly of data: K Yoshimatsu, R Oyama, \\ S Shinohara, T Kuwata; (V) Data analysis and interpretation: K Kuroda, F Tanaka; (VI) Manuscript writing: All authors; (VII) Final approval of \\ manuscript: All authors. \\ Correspondence to: Yusuke Nabe. Second Department of Surgery, School of Medicine, University of Occupational and Environmental Health, 1-1 \\ Iseigaoka, Yahatanishi-ku, Kitakyushu, Fukuoka 807-8555, Japan. Email: y-nabe@med.uoeh-u.ac.jp.
}

Background Approximately $15-29.6 \%$ of patients with thymoma have myasthenia gravis (MG). Some of these patients develop MG after thymectomy despite having no history of MG or related symptoms. Few previous studies have examined the risk factors for the development of post-thymectomy MG in patients with thymoma. Herein, we retrospectively reviewed our institutional experience with patients with thymoma who developed MG after thymectomy.

Methods: Twenty-six patients with thymoma but without MG, who were tested preoperatively for anti-acetylcholine receptor antibody (anti-AChR-Ab) levels, underwent surgical resection at our hospital between 2013 and 2020. Patients with thymic carcinoma were excluded from the study. We evaluated the association of outcomes with preoperative anti-AChR-Ab levels and post-thymectomy MG. We performed a $\chi^{2}$ test for bivariate analysis of categorical data. Differences were considered significant at $\mathrm{P}<0.05$.

Results: The characteristics of the 26 patients (median age: 62 years; 8 men, 18 women) were as follows: World Health Organization (WHO) classifications AB (n=8), B1 (n=9), B2 (n=6), B3 (n=1), and others $(n=2)$ and Masaoka stage I (n=12), II (n=9), III ( $n=3)$, and IVa $(n=2)$. Among the 26 patients, only five had high $(>0.3 \mathrm{nmol} / \mathrm{L})$ preoperative anti-AChR-Ab levels. Post-thymectomy MG occurred in two of the five patients (40\%) with high preoperative anti-AChR-Ab levels. A high preoperative serum anti-AChR-Ab titer was significantly associated with post-thymectomy MG $(\mathrm{P}=0.0267)$. The anti-AChR-Ab titer was also measured postoperatively in four of the five $(80 \%)$ patients with high preoperative levels. The anti-AChR-Ab titer decreased in two of these four patients, and neither developed postoperative MG.

Conclusions: Preoperative and postoperative anti-AChR-Ab positivity might be associated with postthymectomy MG. Therefore, regular measurement of anti-AChR-Ab levels after thymectomy is required.

Keywords: Thymoma; myasthenia gravis (MG); anti-acetylcholine receptor antibody (anti-AChR-Ab); surgery

Submitted Apr 29, 2021. Accepted for publication Jul 16, 2021.

doi: $10.21037 /$ gs-21-287

View this article at: https://dx.doi.org/10.21037/gs-21-287

(c) Gland Surgery. All rights reserved. 


\section{Introduction}

Thymomas occur in $10-12 \%$ of patients with myasthenia gravis (MG) (1), and 15-29.6\% of patients with thymoma have MG $(1,2)$. MG is an autoimmune disease. Its clinical features are attributed to the production of autoimmune antibodies against the acetylcholine receptor at the neuromuscular junction (3). MG presents as weakness of the voluntary ocular, bulbar, and limb muscles; in severe cases, it can lead to death from breathing difficulties (4). Thus, perioperative management of patients with thymoma associated with MG is crucial. Xue et al. reported that $10 \%$ of patients with thymoma and MG experienced a postthymectomy myasthenic crisis (5), whereas other patients developed post-thymectomy MG.

Few previous studies have examined the risk factors for the development of post-thymectomy MG in patients with thymoma. Therefore, we retrospectively reviewed our institutional experience with patients with thymoma who developed MG after thymectomy.

This study is cohort study. We present the following article in accordance with the STROBE reporting checklist (available at https://dx.doi.org/10.21037/gs-21-287).

\section{Methods}

\section{Patients}

We conducted a retrospective review of the medical charts of patients who had been diagnosed as having thymoma and had undergone surgical resection at the Department of Thoracic Surgery at the University of Occupational and Environmental Health (Japan), between April 1st, 2013 and March 31st, 2020.

We extracted data from 26 patients with no history of MG at the time of surgery; those with thymic carcinoma were excluded. We evaluated the association of outcomes with preoperative anti-acetylcholine receptor antibody (anti-AChR-Ab) levels and post-thymectomy MG. MG was diagnosed using multiple tests, including a serum anti-AChR-Ab test, an edrophonium test, a single-fiber electromyography test, and a Harvey-Masland test. Among the patients with high preoperative anti-AChR-Ab levels, one patient developed MG after the surgery. We investigated the association between preoperative anti-AChR-Ab levels and post-thymectomy MG in the hope that it would be useful for future postoperative follow-up of thymoma.

The study was conducted in accordance with the Declaration of Helsinki (as revised in 2013). The study was approved by the ethics committee of the University of Occupational and Environmental Health (Japan; NO.: H26-15), and individual consent for this retrospective analysis was waived.

\section{Anti-ACbR-Ab titer}

At our institution, anti-AChR-Ab levels are routinely measured preoperatively for mediastinal tumors suspected to be thymomas. In cases with high $\mathrm{AChR}-\mathrm{Ab}$ levels before surgery, the treating doctor decides whether the anti-AChR-Ab level needs to be measured regularly after surgery. Measurement of anti-AChR-Ab levels is outsourced to BML which was a Laboratory testing service (including endocrine, plasma protein, biochemical, viral, immunological, hematological, bacteriological, and histopathological tests) and related services, and performed using radioimmunoassay (RIA). 'Cosmic' II was used as the test reagent.

\section{Statistical analysis}

We chose the $\chi^{2}$ test for the bivariate analysis of categorical data as a statistical tool to clarify the association between MG and preoperative anti-AChR-Ab levels. Differences were considered significant at $\mathrm{P}<0.05$. All statistical analyses were carried out using EZR (Saitama Medical Center, Jichi Medical University, Saitama, Japan), a graphical user interface for R (The R Foundation for Statistical Computing, Vienna, Austria).

\section{Results}

A flow diagram of the patient population is shown in Figure 1. The 26 patients with thymomas but without preoperative MG (median age: 62 years; 8 men and 18 women) had the following clinical characteristics (Table 1): World Health Organization classifications of $\mathrm{AB}(\mathrm{n}=8), \mathrm{B} 1$ $(\mathrm{n}=9)$, B2 $(\mathrm{n}=6)$, B3 $(\mathrm{n}=1)$, and others $(\mathrm{n}=2)$ and Masaoka stage I ( $n=12)$, II ( $n=9)$, III $(n=3)$, and IVa $(n=2)$. Complete resection was achieved in all the patients. Three patients $(11.5 \%)$ received postoperative therapy. Of these, two received postoperative radiation therapy and one received intrathoracic cisplatin after surgery. One patient showed thymoma recurrence but did not develop postoperative MG. We encountered a rare case of thymoma associated with pleural dissemination for which extended pleurectomy decortication was performed. This case did 


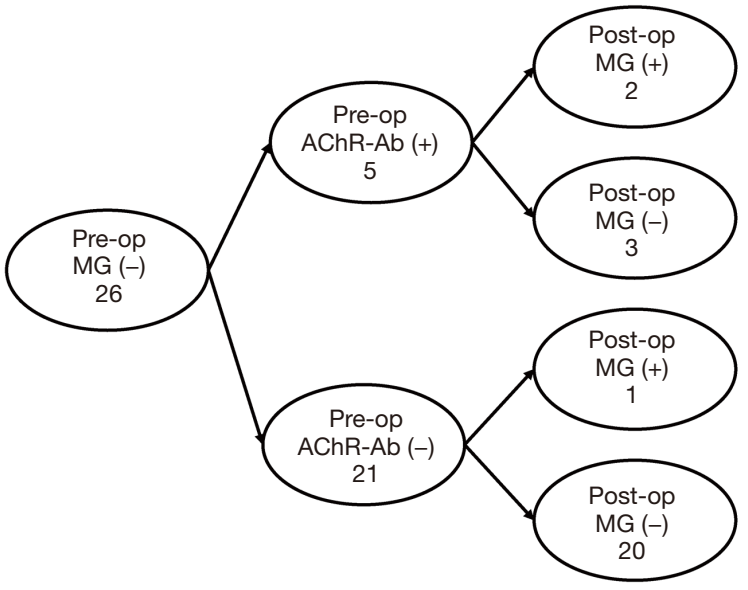

Figure 1 Study flow diagram of the patient population. MG, myasthenia gravis; AChR-Ab, anti-acetylcholine receptor antibody.

not recur for three years after surgery. We have reported this case previously (6).

A summary of patients with high preoperative antiAChR-Ab levels is shown in Table 2. The patient in Case 1 had symptoms of suspected MG with high anti-AChR-Ab levels and was referred to a neurologist. The neurologist performed the Harvey-Masland and Tensilon tests to diagnose MG. The patient in Case 2 was diagnosed as having MG at another institution; hence, we could not collect data on the post-surgery anti-AChR-Ab titers and the day of MG onset. These two cases had no postoperative recurrence of thymomas.

In our study, post-thymectomy MG developed in two of five patients (40\%) with high preoperative anti-AChR$\mathrm{Ab}$ levels. A high preoperative serum anti-AChR-Ab titer was significantly associated with post-thymectomy MG ( $\mathrm{P}=0.0267, \chi^{2}$ test). The positive predictive value of preoperative serum anti-AChR-Ab titers $(>0.3 \mathrm{nmol} / \mathrm{L})$ for developing post-thymectomy MG was $40 \%$, and the negative predictive value was $95.2 \%$, with a sensitivity was $66.7 \%$, and specificity was $87 \%$ (Table 3).

In our study, anti-AChR-Ab was measured postoperatively in four of five $(80 \%)$ patients with preoperative anti-AChR$\mathrm{Ab}$ positivity. In the remaining two patients, anti-AChR$\mathrm{Ab}$ measurements were stopped, and they underwent regular clinical observations instead [median anti-AChR-Ab observation period: 519 days (10-1,028 days)].

Anti-AChR-Ab levels increased in two of the four patients postoperatively compared to those preoperatively,
Table 1 Characteristics of the operative patients

\begin{tabular}{|c|c|}
\hline Complete resection & Value \\
\hline \multicolumn{2}{|l|}{ Age (years) } \\
\hline Median [range] & 62 [24-86] \\
\hline \multicolumn{2}{|l|}{ Gender } \\
\hline Male & 8 \\
\hline Female & 18 \\
\hline \multicolumn{2}{|l|}{ Type of surgery } \\
\hline Tumor resection & 9 \\
\hline Thymothymectomy & 10 \\
\hline Extended thymectomy & 6 \\
\hline Extended pleurectomy decortication & 1 \\
\hline Complete resection & 26 \\
\hline \multicolumn{2}{|l|}{ WHO classification } \\
\hline$A B$ & 8 \\
\hline B1 & 9 \\
\hline B2 & 6 \\
\hline B3 & 1 \\
\hline The other ${ }^{\S}$ & 2 \\
\hline \multicolumn{2}{|l|}{ Masaoka stage } \\
\hline 1 & 12 \\
\hline II & 9 \\
\hline III & 3 \\
\hline IV & 2 \\
\hline \multicolumn{2}{|l|}{ Preoperative AChR-Ab titer (nmol/L) } \\
\hline$>0.3$ & 5 \\
\hline$\leq 0.3$ & 21 \\
\hline Additional treatment & 3 \\
\hline Recurrence & 1 \\
\hline Survival & 26 \\
\hline Observation period (months) & 37.5 [8-89] \\
\hline
\end{tabular}

$\S$, one patient was diagnosed thymoma type B2 and type B3, the other patient was diagnosed type B1 focal type B2. WHO, World Health Organization; AChR-Ab, acetylcholine receptor antibody.

and one of the patients (50\%) was diagnosed as having MG. In contrast, anti-AChR-Ab levels decreased in two of the four patients, and neither developed postoperative MG. 
Table 2 Summary of the operative patients

\begin{tabular}{|c|c|c|c|c|c|c|c|c|c|c|}
\hline Case & $\begin{array}{c}\text { Age } \\
\text { (years old) }\end{array}$ & Gender & Type of surgery & $\begin{array}{c}\text { WHO } \\
\text { classification }\end{array}$ & $\begin{array}{l}\text { Masaoka } \\
\text { stage }\end{array}$ & $\begin{array}{c}\text { Pre-op } \\
\text { AChR-Ab } \\
\text { titer (nmol/L) }\end{array}$ & $\begin{array}{c}\text { Post-op } \\
\text { AChR-Ab } \\
\text { titer (nmol/L) }\end{array}$ & $\begin{array}{c}\text { At Onset } \\
\text { AChR-Ab } \\
\text { titer (nmol/L) }\end{array}$ & $\begin{array}{c}\text { Interval } \\
\text { (days) }\end{array}$ & $\begin{array}{l}\text { AChR-Ab } \\
\text { observation } \\
\text { period } \\
\text { (days) }\end{array}$ \\
\hline 1 & 53 & $\mathrm{~F}$ & Extended thymectomy & Type AB & ॥ & 9.2 & 10.7 & 10.7 & 18 & 668 \\
\hline $2^{\S}$ & 57 & $\mathrm{~F}$ & Thymothymectomy & Type B2 & I & 0.4 & - & - & - & - \\
\hline 4 & 56 & M & Tumor resection & Type AB & I & 0.8 & 2.1 & - & - & 1,028 \\
\hline 5 & 69 & $\mathrm{~F}$ & Extended thymectomy & Type B1 & $\|$ & 16 & 14 & - & - & 10 \\
\hline
\end{tabular}

§, diagnosed MG another institution; hence, we could not collect data on the MG onset day. WHO, World Health Organization; AChR-Ab, acetylcholine receptor antibody; MG, myasthenia gravis.

Table 3 Relationship between occurrence of postoperative MG and preoperative serum titer of anti-AChR-Ab

\begin{tabular}{lccc}
\hline MG status & Pre-op AChR-Ab + & Pre-op AChR-Ab - & Total \\
\hline Post-op MG + & 2 & 1 & 3 \\
Post-op MG - & 3 & 20 & 23 \\
Total & 5 & 21 & 26 \\
\hline
\end{tabular}

MG, myasthenia gravis; anti-AChR-Ab, anti-acetylcholine receptor antibody.

\section{Discussion}

Thymomas are generally thought to exhibit immunological activity and are associated with a variety of autoimmune diseases, including MG. The principle of treatment for thymoma is surgical resection. Extended thymectomy also involves removal of fat around the thymus. The thymus, an organ critical to the immune response, is also a source of disease-causing AChR autoantibody-producing B cells. Nicotinic acetylcholine receptor autoantibodies were identified in the sera of $>85 \%$ of patients with MG (7). Thymectomy results in the suppression of both cellular and humoral immunity and a reduction in anti-AChR-Ab levels, which further leads to sustained improvement and remission of clinical symptoms in patients with MG (8).

MG and AChR are closely related, and this study was designed to determine whether preoperative AChR is associated with post-thymectomy MG. This was a singlecenter study with a few cases, which limited our ability to conduct comparative studies.

Some patients with thymoma develop post-thymectomy MG. Previous reports have shown that MG developed after total thymectomy in $1.5-28 \%$ of thymoma cases (9). In our study, 3 of $26(11.5 \%)$ patients with thymomas but without preoperative MG developed MG after surgery.

The mechanism underlying the onset of postoperative MG remains unclear. Several studies have suggested that thymomas actively export several mature $\mathrm{T}$ cells into peripheral blood. Following this export, the cells persist in the periphery, potentially stimulating autoantibody production and subsequent autoimmune disease $(10,11)$.

Jiang et al. reported that thymus-associated B cell clones were detected in the circulation by both mRNAbased and genomic DNA-based sequencing, and these antigen-experienced $\mathrm{B}$ cells persisted in the circulation after thymectomy (12).

Complete resection was achieved in all the cases in this study. Two of the three patients with post-thymectomy MG underwent extended thymectomy, and the fat around the thymus was removed. Nevertheless, we observed patients who developed MG after surgery. Although we did not investigate the cause of MG after thymoma surgery in this study, circulating $\mathrm{T}$ and $\mathrm{B}$ cells were thought to be involved in the development of MG after thymectomy, as described earlier.

MG complicates the perioperative management after thymoma surgery. The disease can also be fatal if treatment is delayed. Knowledge of the risk of developing postoperative 
Table 4 A review of the literature

\begin{tabular}{lccccl}
\hline $\begin{array}{l}\text { Ref. } \\
\text { number }\end{array}$ & $\begin{array}{c}\text { Number of } \\
\text { patients }\end{array}$ & $\begin{array}{c}\text { Pre-op MG }- \\
\text { thymoma }\end{array}$ & Pre-op AChR-Ab + & Post-op MG + & Risk factors \\
\hline$(1)$ & 229 & 123 & 33 & 10 & Serum AChR-Ab \\
& 104 & 104 & 32 & 8 & Type B1/B2/B3 thymoma history \\
& 67 & 55 & 13 & 5 & High level serum AChR-Ab \\
$(13)$ & 398 & 44 & 7 & 10 & Analyzing: anti-AChR-antibodies is recommended \\
\hline$(14)$ & $15)$ & &
\end{tabular}

MG, myasthenia gravis; anti-AChR-Ab, anti-acetylcholine receptor antibody.

MG before surgery is essential for perioperative management and postoperative follow-up. Therefore, we focused on the relationship between MG and anti-AChR-Ab levels.

The clinical characteristics of patients with postoperative MG from the three studies are shown in Table 4 (1,13-15). Yamada et al. reported that in addition to serum anti-AChR-Ab levels, a history of type B1/B2/B3 thymoma and incomplete resection were risk factors for post-thymectomy MG (1). Mineo et al. and Nakajima et al. reported that only a high antiAChR-Ab titer was a risk factor for post-thymectomy MG $(13,14)$. Marcuse et al. recommended analyzing anti-AChR-Ab levels (15).

In our study, although we were unable to identify an association between the pathological type or incomplete resection and postoperative $M G$, a positive preoperative anti-AChR-Ab level was considered a risk factor for the development of post-thymectomy MG. Further, we found that patients with high preoperative anti-AChR$\mathrm{Ab}$ levels who showed a decrease in anti-AChR-Ab levels postoperatively did not develop MG postoperatively. Thus, we suggest that not only positive preoperative $\mathrm{AChR}-\mathrm{Ab}$ levels but also changes in postoperative AChR$\mathrm{Ab}$ levels may be associated with post-thymectomy MG. Marcuse et al. reported that at least $91 \%$ of patients with subclinical MG developed clinical MG within 6 years after thymectomy (15). Unfortunately, there is no literature reporting on AChR-Ab levels after surgery. Therefore, regular anti-AChR-Ab measurements after thymectomy should be performed to further investigate the association between postoperative anti-AChR-Ab levels and MG.

The limitations of this study are its small sample size and its single-center retrospective design. Because small sample sizes lead to limited statistical power, evidence on using anti-AChR-Ab levels as a predictor of MG after thymectomy is limited. Therefore, our study results may not be generalizable. In the future, our study can impact perioperative management beyond preoperative prediction of the onset of postoperative MG. For postoperative cases of thymoma with high anti-AChR-Ab levels before surgery, determining whether the anti-AChR-Ab level needs to be measured on a regular basis after surgery was per the discretion of each attending physician. Our results suggest that in the future it may be necessary to measure antiAChR-Ab levels on a regular basis after surgery.

\section{Conclusions}

Preoperative and postoperative AChR-Ab positivity might be associated with post-thymectomy MG. In particular, postoperative anti-AChR antibody decline may be a good prognostic factor about the onset of MG after surgery. Therefore, regular anti-AChR-Ab measurement after thymectomy is required.

\section{Acknowledgments}

We would like to thank Editage (www.editage.com) for English language editing.

Funding: None.

\section{Footnote}

Reporting Checklist: The authors have completed the STROBE reporting checklist. Available at https://dx.doi. org/10.21037/gs-21-287

Data Sharing Statement: Available at https://dx.doi. org/10.21037/gs-21-287 
Peer Review File: Available at https://dx.doi.org/10.21037/ gs-21-287

Conflicts of Interest: All authors have completed the ICMJE uniform disclosure form (available at https://dx.doi. org/10.21037/gs-21-287). The authors have no conflicts of interest to declare.

Ethical Statement: The authors are accountable for all aspects of the work in ensuring that questions related to the accuracy or integrity of any part of the work are appropriately investigated and resolved. The study was conducted in accordance with the Declaration of Helsinki (as revised in 2013). The study was approved by the ethics committee of the University of Occupational and Environmental Health (Japan; No. H26-15), and individual consent for this retrospective analysis was waived.

Open Access Statement: This is an Open Access article distributed in accordance with the Creative Commons Attribution-NonCommercial-NoDerivs 4.0 International License (CC BY-NC-ND 4.0), which permits the noncommercial replication and distribution of the article with the strict proviso that no changes or edits are made and the original work is properly cited (including links to both the formal publication through the relevant DOI and the license). See: https://creativecommons.org/licenses/by-nc-nd/4.0/.

\section{References}

1. Yamada Y, Yoshida S, Iwata T, et al. Risk factors for developing postthymectomy myasthenia gravis in thymoma patients. Ann Thorac Surg 2015;99:1013-9.

2. Shen J, Tie H, Xu A, et al. Inter-relationship among myasthenia gravis, WHO histology, and Masaoka clinical stage and effect on surgical methods in patients with thymoma: a retrospective cohort study. J Thorac Dis 2018;10:2981-90.

3. Kondo K, Monden Y. Thymoma and myasthenia gravis: a clinical study of 1,089 patients from Japan. Ann Thorac Surg 2005;79:219-24.

4. De Roxas RC, Bagnas MA, Baldonado JJ, et al. Clinical Profile and Outcome of Postthymectomy versus NonThymectomy Myasthenia Gravis Patients in the Philippine General Hospital: A 6-Year Retrospective Study. Front Neurol 2016;7:96.

5. Xue L, Wang L, Dong J, et al. Risk factors of myasthenic crisis after thymectomy for thymoma patients with myasthenia gravis. Eur J Cardiothorac Surg 2017;52:692-7.

6. Imanishi N, Nabe Y, Takenaka M, et al. Extended pleurectomy decortication for thymoma with pleural dissemination. Gen Thorac Cardiovasc Surg 2019;67:814-7.

7. Verschuuren JJ, Huijbers MG, Plomp JJ, et al. Pathophysiology of myasthenia gravis with antibodies to the acetylcholine receptor, muscle-specific kinase and lowdensity lipoprotein receptor-related protein 4 . Autoimmun Rev 2013;12:918-23.

8. Song Y, Zhou L, Miao F, et al. Increased frequency of thymic $\mathrm{T}$ follicular helper cells in myasthenia gravis patients with thymoma. J Thorac Dis 2016;8:314-22.

9. Kondo K, Monden Y. Myasthenia gravis appearing after thymectomy for thymoma. Eur J Cardiothorac Surg 2005;28:22-5.

10. Hoffacker V, Schultz A, Tiesinga JJ, et al. Thymomas alter the T-cell subset composition in the blood: a potential mechanism for thymoma-associated autoimmune disease. Blood 2000;96:3872-9.

11. Buckley C, Douek D, Newsom-Davis J, et al. Mature, long-lived CD4+ and CD8+ T cells are generated by the thymoma in myasthenia gravis. Ann Neurol 2001;50:64-72.

12. Jiang R, Hoehn KB, Lee CS, et al. Thymus-derived B cell clones persist in the circulation after thymectomy in myasthenia gravis. Proc Natl Acad Sci U S A 2020;117:30649-60.

13. Mineo TC, Tamburrini A, Schillaci O, et al. Onset and Evolution of Clinically Apparent Myasthenia Gravis After Resection of Non-myasthenic Thymomas. Semin Thorac Cardiovasc Surg 2018;30:222-7.

14. Nakajima J, Murakawa T, Fukami T, et al. Postthymectomy myasthenia gravis: relationship with thymoma and antiacetylcholine receptor antibody. Ann Thorac Surg 2008;86:941-5.

15. Marcuse F, Hochstenbag M, Hoeijmakers JGJ, et al. Subclinical myasthenia gravis in thymomas. Lung Cancer 2021;152:143-8.

Cite this article as: Nabe Y, Hashimoto T, Tanaka K, Fujita Y, Yoshimatsu K, Nemoto Y, Oyama R, Matsumiya H, Mori M, Kanayama M, Taira A, Shinohara S, Kuwata T, Takenaka M, Tashima Y, Kuroda K, Tanaka F. Relationship between antiacetylcholine receptor antibodies and the development of postthymectomy myasthenia gravis in patients with thymoma: a single-center experience. Gland Surg 2021;10(8):2408-2413. doi: $10.21037 / g s-21-287$ 